\title{
Recent trends in the characteristics and prognosis of patients hospitalized with acute heart failure
}

This article was published in the following Dove Press journal:

Clinical Epidemiology

21 November 201।

Number of times this article has been viewed

\section{David Park' \\ David McManus' \\ Chad Darling ${ }^{3}$ \\ Jordan H Goldberg² \\ Joel M Gore ${ }^{1,2}$ \\ Darleen Lessard ${ }^{2}$ \\ Robert J Goldberg² \\ 'Department of Medicine, University of Massachusetts Medical School, \\ ${ }^{2}$ Division of Epidemiology of Chronic Diseases, Department of Quantitative Health Sciences, University of Massachusetts Medical School, ${ }^{3}$ Department of Emergency Medicine, University of Massachusetts Medical School, Worcester, MA, USA}

Correspondence: Robert J Goldberg Department of Quantitative Health Sciences, University of Massachusetts Medical School, 55 Lake Avenue North, Worcester, MA 01655, USA

Tel + I 508856399 |

Fax + I 5088564596

Email robert.goldberg@umassmed.edu
Background: Despite the magnitude and impact of heart failure (HF) in the United States, relatively little data are available that describe the prognosis associated with acute HF, especially from the perspective of a population-based investigation. The purpose of this nonconcurrent prospective study was to describe the overall, and changing trends therein, prognosis of 4228 patients discharged from all eleven greater Worcester (MA) medical centers after a documented episode of acute HF and factors associated with an increased risk of dying after hospital discharge.

Methods: The study population consisted of residents of the Worcester metropolitan area discharged after being hospitalized for acute HF at all greater Worcester medical centers during $1995(\mathrm{n}=1783)$ and $2000(\mathrm{n}=2445)$.

Results: The 3-month (20\% versus 18\%), 1-year (41\% versus 38\%), and 5-year ( $84 \%$ versus $82 \%$ ) death rates were lower in patients discharged from all metropolitan Worcester hospitals in 2000 versus 1995, respectively. Improving long-term survival rates for patients discharged in 2000 as compared with 1995 were magnified after controlling for several confounding demographic and clinical factors of prognostic importance. A number of potentially modifiable demographic, medical history, and clinical factors were associated with an increased risk of dying during the first year after hospital discharge for acute HF.

Conclusion: The results of this community-wide observational study suggest improving trends in the long-term prognosis after acute HF. Despite these encouraging trends, the long-term prognosis for patients with acute HF remains poor, and several at-risk groups can be identified for early intervention and increased monitoring efforts.

Keywords: population-based study, long-term prognosis, post-discharge survivors

\section{Introduction}

Heart failure (HF) is a major and growing public health and clinical problem in the United States and other industrialized nations. ${ }^{1,2}$ Despite advances in the medical and nonpharmacologic management of patients with acute and chronic manifestations of HF, the prognosis of patients with HF remains poor. ${ }^{3-8}$ Given the aging of the American population, the improved treatment of coronary heart disease and HF, as well as the ongoing epidemics of obesity and diabetes, there is a myriad of reasons to believe that the characteristics and prognosis of persons with HF may have changed over time. Despite the magnitude and impact of this clinical syndrome, however, few contemporary data exist, particularly from the more generalizable perspective of a population-based study, describing the long-term prognosis of patients hospitalized with decompensated HF, changes over time therein, or the independent predictors of 
death among representative populations with acute HF. In a prior study carried out in the metropolitan Worcester population, the long-term prognosis of patients discharged from all greater Worcester medical centers after being hospitalized for acute HF in 2000 and factors associated with an increased risk of dying were examined. ${ }^{8}$

The objectives of the present community-wide observational study were to describe the overall prognosis of patients discharged from the hospital after acute HF, changing trends in the post-discharge survival of these patients, and to identify demographic and clinical factors associated with a poor long-term prognosis. Residents of the Worcester (MA) metropolitan area discharged from all greater Worcester medical centers after an episode of documented acute HF in 1995 and 2000 as part of the Worcester Heart Failure Study comprised the population of this report. .,10 $^{9}$

\section{Methods}

Adult male and female residents of all ages from the Worcester (MA) metropolitan area (2000 census estimate $=478,000)$ hospitalized for possible acute $\mathrm{HF}$ at all eleven greater Worcester medical centers during 1995 and 2000 comprised the study population. For purposes of identifying the study population, the medical records of patients with primary or secondary International Classification of Diseases, Ninth Revision (ICD-9) codes consistent with the possible presence of HF were reviewed in a standardized manner. ${ }^{9,10}$ Patients with a discharge diagnosis of HF (ICD-9 code 428) comprised the primary diagnostic rubric reviewed for the identification of cases of possible HF. In addition, the medical records of patients with discharge diagnoses of rheumatic HF (ICD-9 code 398.9), hypertensive heart and renal disease (ICD-9 codes 402 and 404, respectively), acute cor pulmonale (ICD-9 code 415), other diseases of the endocardium (ICD-9 code 424), cardiomyopathy (ICD-9 code 425.4), pulmonary heart disease and congestion (ICD-9 codes 416.9 and 514, respectively), acute lung edema (ICD-9 code 518.4), edema (ICD-9 code 782.3), and dyspnea and respiratory abnormalities (ICD-9 code 786) were reviewed by trained study physicians and nurses to identify patients who may have had newly diagnosed acute $\mathrm{HF}$.

The diagnosis of acute HF in greater Worcester residents presenting to area hospitals with signs and symptoms of HF was defined as the presence of decompensated HF based on use of the Framingham Study criteria. ${ }^{11}$ These criteria included the presence of two major criteria (eg, rales and distended neck veins) or one major and two minor (eg, night cough and dyspnea on ordinary exertion) criteria.
An incident (first) event of acute HF was defined as the absence of a prior hospitalization for HF, physician diagnosis of HF, or past treatment for HF based on the review of data contained in hospital medical records. Patients who developed HF secondary to admission for another acute illness (eg, acute myocardial infarction) or after a procedure or surgery (eg, percutaneous coronary intervention) were not included. Patients who died during the index hospitalization were excluded from the final analytic sample, since the aim was to describe the long-term prognosis of discharged hospital patients.

\section{Data collection}

Information was collected about patient's demographic, medical history, and clinical characteristics, as well as laboratory test results, through the review of information contained in hospital medical records. This included information about a patient's age, sex, race/ethnicity, body mass index (BMI), initial symptoms of HF, physical examination findings, clinical characteristics, prior comorbidities (eg, stroke, hypertension, diabetes mellitus), and laboratory findings (eg, serum levels of glucose, hematocrit, estimated glomerular filtration rate [eGFR] $).{ }^{12}$ Since ejection fraction findings during the index hospitalization were available for only one-third of hospital survivors of decompensated HF in the present study, this variable was not used in the analysis of factors associated with long-term prognosis after acute HF.

Physicians' progress notes and daily medication logs were reviewed for the prescribing of selected medications. The use of cardiac medications that have been shown to be of benefit in improving the prognosis of patients with HF ( $\beta$-blockers, angiotensin receptor blockers, and angiotensin-converting enzyme inhibitors), as well as the use of medications shown to be effective in relieving the symptoms of patients with acute HF (digoxin and diuretics) were examined. ${ }^{13}$ Information about patients' long-term survival status was obtained through the review of hospital medical records at all participating medical centers for subsequent hospitalizations or medical care contacts, as well as through the review of the Social Security Death Index and statewide death certificates.

\section{Data analysis}

A life-table approach was used to examine long-term mortality patterns after greater Worcester residents were discharged from all metropolitan Worcester medical centers after a first episode of acute HF or a new exacerbation of previously diagnosed HF in 1995 and 2000 through the end of 2008, including 
patients with varying lengths of long-term follow-up. Long-term all-cause post-hospital-discharge death rates were calculated in a standard manner with accompanying 95\% confidence intervals (CIs). Differences in the distribution of selected demographic and clinical characteristics between post-discharge decedents and survivors were examined using chi-square tests and $t$-tests for discrete and continuous variables, respectively. Differences in the long-term prognosis of patients discharged from all greater Worcester hospitals after acute $\mathrm{HF}$ at selected post-discharge time points were examined through the use of chi-square tests and the log-rank test for statistical significance.

A logistic regression approach was used to identify demographic, medical history, laboratory, and clinical factors associated with a poor prognosis after $\mathrm{HF}$ at 3 and 12 months after hospital discharge, given the high risk of dying at these time points. Multivariable adjusted odds ratios (ORs) and accompanying 95\% CIs of factors associated with a poor prognosis at 3 months and at 1 year after hospital discharge were calculated in a standard manner. These periods were chosen for analysis due to the high death rates experienced by patients with acute HF during the first several months to 1 year after hospital discharge for HF. Since further information was not available about changes in patients' clinical or treatment statuses after being discharged from greater Worcester medical centers during the years under study, the identification of factors associated with a poor prognosis was focused on those that could be identified at the time of hospital discharge for HF and that may be amenable to change. The use of various hospital therapies were not controlled for in these multivariable adjusted regression analyses, due to the nonrandomized observational nature of the present noncurrent prospective study and potential for confounding by treatment indication. Further, treatment variables were not included in the multivariable adjusted regression models due to the difficulty in interpretation of any resultant findings.

\section{Results}

\section{Baseline characteristics}

After an extensive review of hospital medical records and various disease diagnostic rubrics in which cases of possible HF might be found, a total of 1949 greater Worcester residents were hospitalized for acute HF in the year 1995 and 2587 in the year 2000; of these, there were a total of 508 incident (initial episode) events of acute HF in 1995 and 645 cases of newly diagnosed acute HF in 2000. In 1995, 8.5\% of all cases of acute HF died during their index hospitalization compared with $5.5 \%$ of all patients with acute HF in 2000; among incident cases of acute HF, $7.9 \%$ died in 1995, whereas $5.7 \%$ died in 2000. After excluding patients who died during their index hospitalization, in order to systematically characterize the post-discharge prognosis of patients with acute HF, the final analytic sample consisted of 1783 hospital survivors in 1995 and 2445 patients in 2000; of these, 468 were incident cases of acute HF in 1995 and 608 in 2000.

The average age of the study population was 76 years, $57 \%$ were women, and approximately three-quarters had been previously diagnosed with HF based on a review of the information contained in hospital medical records. There were no appreciable differences in the average age (75 years versus 76 years), proportion of women (58\% versus $57 \%$ ), or percentage of patients presenting with a first episode of HF (26\% versus $25 \%$ ) between hospital survivors of HF in 1995 as compared with those discharged in 2000 , respectively.

\section{Long-term death rates and changing trends therein}

In examining the long-term prognosis for all patients discharged after a confirmed episode of acute HF, the 3-month, 1-year, and 5-year death rates were $18.8 \%$ (95\% CI $17.6 \%-20.0 \%$ ), 39.7\% (95\% CI $38.2 \%-41.2 \%$ ), and $82.9 \%$ (95\% CI $81.7 \%-84.0 \%)$, respectively.

Patients discharged from all greater Worcester medical centers in 2000 after hospitalization for decompensated HF experienced slightly lower all-cause death rates than patients discharged in 1995 at each of the time points examined, namely 3 months, 1 year, and 5 years (Table 1). When post-discharge death rates in persons with an incident (first) documented episode of acute HF were examined, patients discharged from all greater Worcester hospitals in 2000 also experienced slightly lower post-discharge death rates at 3 months, 1 year, and at 5 years compared with patients discharged in 1995 (Table 1).

A series of multiple regression analyses were carried out for purposes of determining whether the long-term prognosis after HF has changed during the years under study after controlling for a number of factors of prognostic importance. After controlling for age, sex, medical history of several comorbid conditions, acute symptoms, and laboratory findings in these regression analyses, patients discharged from all greater Worcester hospitals in 2000, as compared with those discharged in 1995 , were at considerably lower risk of dying at 3 months (OR $=0.85,95 \%$ CI $0.73-1.00)$ and at 1 year $(\mathrm{OR}=0.81,95 \% \mathrm{CI}=0.71-0.92)$. 
Table I Post-discharge death rates in hospital survivors of acute $\mathrm{HF}$ at selected time points

\begin{tabular}{|c|c|c|c|c|}
\hline \multirow{3}{*}{$\begin{array}{l}\text { Post-discharge } \\
\text { follow-up }\end{array}$} & \multicolumn{4}{|c|}{ Death rate, \% $(95 \% \mathrm{Cl})$} \\
\hline & \multicolumn{2}{|l|}{ All patients } & \multicolumn{2}{|c|}{$\begin{array}{l}\text { Incident cases } \\
\text { of acute HF }\end{array}$} \\
\hline & 1995 & 2000 & 1995 & 2000 \\
\hline 3 months & $\begin{array}{l}19.9 \% \\
(18.1-21.8)\end{array}$ & $\begin{array}{l}18.0 \% \\
(16.5-19.6)\end{array}$ & $\begin{array}{l}15.8 \% \\
(12.8-19.4)\end{array}$ & $\begin{array}{l}13.8 \% \\
(11.3-16.8)\end{array}$ \\
\hline I year & $\begin{array}{l}41.4 \% \\
(39.2-43.8)\end{array}$ & $\begin{array}{l}38.4 \% \\
(36.5-40.3)\end{array}$ & $\begin{array}{l}29.1 \% \\
(25.2-33.4)\end{array}$ & $\begin{array}{l}28.9 \% \\
(25.5,32.7)\end{array}$ \\
\hline 5 years & $\begin{array}{l}84.2 \% \\
(82.5-85.9)\end{array}$ & $\begin{array}{l}81.9 \% \\
(80.4-83.4)\end{array}$ & $\begin{array}{l}72.2 \% \\
(68.1-76.2)\end{array}$ & $\begin{array}{l}66.6 \% \\
(62.9-70.3)\end{array}$ \\
\hline
\end{tabular}

Abbreviations: $\mathrm{HF}$, heart failure; $\mathrm{Cl}$, confidence interval.

\section{Factors associated with 3 month and I year death rates}

Given the high death rates experienced by hospital survivors of acute HF during the first year after being discharged from the hospital after acute decompensated HF, the demographic and clinical characteristics of post-discharge decedents were compared with survivors at 3 months and at 1 year (Table 2).

Overall, patients who died during the first 3 months after hospital discharge were generally older, had a lower BMI, and were more likely to have been previously diagnosed with anemia, chronic kidney disease, stroke, and HF than survivors of this high-risk period (Table 2). Patients who died during the first 3 months following hospital discharge were more likely to present with weakness at the time of their index hospitalization than post-discharge survivors. Patients dying during the first 3 months after hospital discharge had markedly lower blood pressure findings than patients who survived this period. Post-discharge decedents were less likely to have been prescribed angiotensin converting enzyme (ACE) inhibitors/angiotensin receptor blockers, aspirin, beta blockers, and digoxin than patients who survived the initial 3 months or 1 year after hospital discharge. Relatively similar characteristics were associated with an adverse prognosis during the first 3 months and 1 year after hospital discharge for HF when the characteristics of postdischarge decedents were examined, comparing survivors hospitalized in 1995 with those hospitalized in 2000 (data not shown).

Similarly, when the characteristics of individuals hospitalized with acute HF who died during the first year after hospital discharge were compared with those who did not die over this period, post-discharge decedents were older, had a lower BMI, and were more likely to have had anemia, chronic obstructive pulmonary disease (COPD), or HF previously diagnosed (Table 2). Persons who died during the first year after hospital discharge had a different symptom profile at the time of hospital admission than persons who survived this period. Decedents in the first year after hospital discharge were also less likely than survivors to have been treated with effective cardiac therapies. A relatively similar profile of demographic, clinical, and treatment characteristics was observed in 1 -year post-discharge decedents as compared with postdischarge survivors during each of the 2 years under study (data not shown).

A series of multivariable adjusted regression analyses were carried out for purposes of identifying factors independently associated with post-discharge death rates at 3 months and 1 year, overall and separately, in the two patient cohorts (Table 3).

Advanced age, a history of COPD or stroke, generalized weakness at the time of hospital presentation, and lower eGFR and systolic blood pressure findings were associated with an increased odds of dying during the first 3 months after hospital discharge for decompensated HF. On the other hand, a higher BMI and a history of hypertension were associated with a lower odds of dying within 3 months after hospital discharge. Relatively similar prognostic factors were associated with an adverse prognosis in each of the two study cohorts (1995 and 2000), though their predictive importance varied slightly.

Similar factors to those identified as being of prognostic importance during the first 3 months after hospital discharge were associated with an increased risk for dying during the first year after hospital discharge (Table 3 ). In addition, a history of previously diagnosed anemia, chronic kidney disease, and HF were associated with a greater likelihood of dying at 1 year; the presence of orthopnea at the time of hospital admission was associated with a lower odds of dying at 1 year after hospital discharge for HF. Relatively similar factors were associated with an increased risk of dying during the first year after hospital discharge in patients hospitalized in 1995 and in those hospitalized during 2000.

\section{Discussion}

The results of this large observational study in residents of metropolitan Worcester hospitalized at all greater Worcester medical centers with acute HF demonstrate that patients with this clinical syndrome experience high death rates after hospital discharge. Despite the overall poor prognosis of this condition, however, there were slight improvements in the crude 3-month and 1-year survival rates noted between 
Table 2 Characteristics of post-discharge survivors and decedents of acute heart failure

\begin{tabular}{|c|c|c|c|c|c|c|}
\hline \multirow[t]{2}{*}{ Characteristic } & \multicolumn{3}{|c|}{3 months after hospital discharge } & \multicolumn{3}{|c|}{ I year after hospital discharge } \\
\hline & $\begin{array}{l}\text { Survivors } \\
(n=3439)\end{array}$ & $\begin{array}{l}\text { Decedents } \\
(\mathrm{n}=789)\end{array}$ & $P$-value & $\begin{array}{l}\text { Survivors } \\
(n=255 I)\end{array}$ & $\begin{array}{l}\text { Decedents } \\
(n=1677)\end{array}$ & $P$-value \\
\hline Age (mean, years) & 75.1 & 78.7 & $<0.001$ & 75.1 & 78.7 & $<0.001$ \\
\hline Age (years) & n (\%) & n (\%) & & n (\%) & n (\%) & \\
\hline$<65$ & $586(17.1)$ & $68(8.6)$ & & $474(18.6)$ & $180(10.7)$ & \\
\hline $65-74$ & $826(24.0)$ & $157(19.9)$ & $<0.001$ & $641(25.2)$ & $342(20.4)$ & $<0.001$ \\
\hline $75-84$ & I27| (37.0) & $319(40.4)$ & & $925(36.3)$ & $665(39.7)$ & \\
\hline$\geq 85$ & $754(21.9)$ & $245(31.1)$ & & $509(20.0)$ & $490(29.2)$ & \\
\hline Male (\%) & $1477(43.0)$ & $343(43.5)$ & 0.82 & $1069(41.9)$ & 75 I (44.8) & 0.06 \\
\hline White race (\%) & $3207(93.3)$ & $764(96.8)$ & $<0.001$ & $2356(92.4)$ & $1615(96.3)$ & $<0.001$ \\
\hline \multicolumn{7}{|l|}{ Body mass index (\%) } \\
\hline$<25$ & $1200(4 \mid .8)$ & $364(58.2)$ & & $821(38.5)$ & $743(54.6)$ & \\
\hline $25.0-29.9$ & $803(28.0)$ & $145(23.2)$ & $<0.001$ & $614(28.8)$ & $334(24.5)$ & $<0.001$ \\
\hline$\geq 30$ & $868(30.2)$ & II7 (I8.7) & & $700(32.8)$ & $285(20.9)$ & \\
\hline \multicolumn{7}{|l|}{ Medical history (\%) } \\
\hline Anemia & $760(22.1)$ & $225(28.5)$ & $<0.001$ & $490(19.2)$ & $495(29.5)$ & $<0.001$ \\
\hline Coronary heart disease & |97| (57.3) & $452(57.3)$ & 0.99 & $1432(56.1)$ & 991 (59.1) & 0.06 \\
\hline COPD & $1172(34.1)$ & $297(37.6)$ & 0.06 & $832(32.6)$ & $637(38.0)$ & $<0.005$ \\
\hline Diabetes & $1400(40.7)$ & $309(39.2)$ & 0.45 & $1023(40.1)$ & $686(40.9)$ & 0.60 \\
\hline Hypertension & $2303(67.0)$ & $478(60.6)$ & $<0.001$ & $1722(67.5)$ & $1,059(63.2)$ & $<0.005$ \\
\hline Peripheral vascular disease & $635(18.5)$ & I38 (I7.5) & 0.56 & $445(17.4)$ & $328(19.6)$ & 0.09 \\
\hline Chronic kidney disease & $762(22.2)$ & $223(28.3)$ & $<0.001$ & $489(19.2)$ & $496(29.6)$ & $<0.001$ \\
\hline Stroke & $470(13.7)$ & $146(\mid 8.5)$ & $<0.005$ & $353(13.8)$ & $263(15.7)$ & 0.11 \\
\hline Heart failure & $2521(73.3)$ & $631(80.0)$ & $<0.001$ & $1787(70.1)$ & $1365(81.4)$ & $<0.001$ \\
\hline \multicolumn{7}{|l|}{ Presenting symptoms } \\
\hline Chest pain & $1135(33.0)$ & $204(25.9)$ & $<0.001$ & $913(35.8)$ & $426(25.4)$ & $<0.001$ \\
\hline Dyspnea & $326 I(94.8)$ & 74I (93.9) & 0.35 & $2428(95.2)$ & 1574 (93.9) & 0.07 \\
\hline Edema & $2319(67.4)$ & $542(68.7)$ & 0.52 & $1686(66.1)$ & $1175(70.1)$ & $<0.01$ \\
\hline Nausea/vomiting & $520(15.1)$ & $102(12.9)$ & $<0.13$ & $399(15.6)$ & $223(13.3)$ & $<0.05$ \\
\hline Orthopnea & $1232(35.8)$ & $229(29.0)$ & $<0.001$ & $948(37.2)$ & $513(30.6)$ & $<0.001$ \\
\hline Weakness & $84 \mid(24.5)$ & $270(34.2)$ & $<0.001$ & $596(23.4)$ & $515(30.7)$ & $<0.001$ \\
\hline Weight gain & $293(8.5)$ & $61(7.7)$ & 0.52 & $204(8.0)$ & $150(8.9)$ & 0.28 \\
\hline \multicolumn{7}{|l|}{ Laboratory findings (mean, mg/dL) } \\
\hline Glucose & $3396(166.5)$ & $777(164.3)$ & 0.50 & $2519(167.7)$ & 1654 (163.7) & 0.50 \\
\hline Estimated GFR & $3398(64.7)$ & $775(58.1)$ & $<0.001$ & $2517(66.6)$ & $1656(58.7)$ & $<0.001$ \\
\hline Hematocrit & 3391 (36.7) & $780(35.7)$ & $<0.01$ & & & \\
\hline Physiologic findings & & & & $2516(36.9)$ & $1655(35.8)$ & $<0.001$ \\
\hline Systolic blood pressure (mean, mmHg) & $3432(148.3)$ & $784(133.9)$ & $<0.001$ & $2544(150.1)$ & $1672(138.8)$ & $<0.001$ \\
\hline Diastolic blood pressure (mean, $\mathrm{mmHg}$ ) & $3396(78.4)$ & $769(72.3)$ & $<0.001$ & $2517(79.2)$ & I 648 (74.2) & $<0.001$ \\
\hline Heart rate (bpm) & $3436(90.6)$ & $788(90.0)$ & 0.49 & $2548(90.8)$ & $1676(89.8)$ & 0.16 \\
\hline \multicolumn{7}{|l|}{ Medications } \\
\hline ACE inhibitors/ARBs & 2025 (58.9) & $330(4 I .8)$ & $<0.00$ I & $1527(59.8)$ & $828(49.4)$ & $<0.001$ \\
\hline Aspirin & $1788(52.0)$ & $343(43.5)$ & $<0.00$ I & $1355(53.1)$ & $776(46.3)$ & $<0.001$ \\
\hline Beta blockers & $1512(44.0)$ & $260(33.0)$ & $<0.001$ & I I 68 (45.8) & $604(36.0)$ & $<0.001$ \\
\hline Digoxin & I70I (49.5) & $442(56.0)$ & $<0.001$ & $1203(47.2)$ & $940(56.1)$ & $<0.001$ \\
\hline Diuretics & $3361(97.7)$ & $778(98.6)$ & 0.16 & $2488(97.5)$ & I65I (98.5) & 0.05 \\
\hline
\end{tabular}

Abbreviations: ACE, angiotensin converting enzyme; ARB, angiotensin receptor blocker; COPD, chronic obstructive pulmonary disease; GFR, glomerular filtration rate.

1995 and 2000. These findings were further magnified after controlling for a number of demographic and clinical factors of long-term prognostic importance. A number of demographic and clinical characteristics were able to be identifed that were associated with an increased risk for dying during the first year after hospital discharge for acute HF.

\section{Prognosis after HF}

Residents of greater Worcester with acute HF in 1995 and 2000 experienced high death rates after hospital discharge. Indeed, hospitalization for acute HF has been shown to be one of the strongest risk factors for dying in patients with a history of HF. ${ }^{14}$ On the other hand, the results of the present 
Table 3 Factors significantly associated with higher death rates at 3 months and I year after hospital discharge for acute heart failure

\begin{tabular}{|c|c|c|c|c|c|c|}
\hline \multirow[t]{3}{*}{ Characteristic } & \multicolumn{6}{|l|}{ OR $(95 \% \mathrm{Cl})$} \\
\hline & \multicolumn{3}{|l|}{3 months } & \multicolumn{3}{|l|}{ I year } \\
\hline & Total population & 1995 cohort & 2000 cohort & Total population & 1995 cohort & 2000 cohort \\
\hline \multicolumn{7}{|l|}{ Age (years) } \\
\hline $65-74$ & $\begin{array}{l}1.42 \\
(1.03-1.95)\end{array}$ & $\begin{array}{l}0.99 \\
(0.62-1.56)\end{array}$ & $\begin{array}{l}1.92 \\
(1.21-3.05)\end{array}$ & $\begin{array}{l}1.20 \\
(0.95-1.5 I)\end{array}$ & $\begin{array}{l}\text { I.II } \\
(0.78-1.56)\end{array}$ & $\begin{array}{l}1.25 \\
(0.90-1.74)\end{array}$ \\
\hline $75-84$ & $\begin{array}{l}1.86 \\
(1.38-2.51)\end{array}$ & $\begin{array}{l}1.58 \\
(1.03-2.42)\end{array}$ & $\begin{array}{l}2.09 \\
(1.35-3.24)\end{array}$ & $\begin{array}{l}1.62 \\
(1.30-2.01)\end{array}$ & $\begin{array}{l}1.46 \\
(1.05-2.04)\end{array}$ & $\begin{array}{l}1.78 \\
(1.32-2.4 I)\end{array}$ \\
\hline$\geq 85$ & $\begin{array}{l}2.28 \\
(1.66-3.13)\end{array}$ & $\begin{array}{l}1.94 \\
(1.22-3.08)\end{array}$ & $\begin{array}{l}2.73 \\
(1.74-4.29)\end{array}$ & $\begin{array}{l}2.14 \\
(1.69-2.72)\end{array}$ & $\begin{array}{l}1.78 \\
(1.23-2.57)\end{array}$ & $\begin{array}{l}2.55 \\
(1.85-3.53)\end{array}$ \\
\hline \multicolumn{7}{|l|}{ BMI } \\
\hline $25.0-29.9$ & $\begin{array}{l}0.68 \\
(0.55-0.85)\end{array}$ & $\begin{array}{l}0.58 \\
(0.4 I-0.82)\end{array}$ & $\begin{array}{l}0.77 \\
(0.59-1.02)\end{array}$ & $\begin{array}{l}0.71 \\
(0.60-0.84)\end{array}$ & $\begin{array}{l}0.56 \\
(0.43-0.73)\end{array}$ & $\begin{array}{l}0.83 \\
(0.66-1.03)\end{array}$ \\
\hline$\geq 30$ & $\begin{array}{l}0.58 \\
(0.46-0.73)\end{array}$ & $\begin{array}{l}0.60 \\
(0.42-0.86)\end{array}$ & $\begin{array}{l}0.58 \\
(0.42-0.79)\end{array}$ & $\begin{array}{l}0.6 \mathrm{I} \\
(0.5 \mathrm{I}-0.72)\end{array}$ & $\begin{array}{l}0.60 \\
(0.45-0.78)\end{array}$ & $\begin{array}{l}0.63 \\
(0.50-0.80)\end{array}$ \\
\hline \multicolumn{7}{|l|}{ Medical History } \\
\hline Anemia & $\begin{array}{l}1.12 \\
(0.91-1.37)\end{array}$ & $\begin{array}{l}1.04 \\
(0.76-1.42)\end{array}$ & $\begin{array}{l}1.16 \\
(0.88-1.52)\end{array}$ & $\begin{array}{l}1.34 \\
(1.14-1.59)\end{array}$ & $\begin{array}{l}1.32 \\
(1.02-1.72)\end{array}$ & $\begin{array}{l}1.35 \\
(1.08-1.68)\end{array}$ \\
\hline COPD & $\begin{array}{l}1.22 \\
(1.03-1.46)\end{array}$ & $\begin{array}{l}1.31 \\
(1.00-1.70)\end{array}$ & $\begin{array}{l}1.16 \\
(0.92-1.47)\end{array}$ & $\begin{array}{l}1.31 \\
(1.13-1.50)\end{array}$ & $\begin{array}{l}I .21 \\
(0.97-\mid .5 I)\end{array}$ & $\begin{array}{l}1.41 \\
(1.17-1.70)\end{array}$ \\
\hline Hypertension & $\begin{array}{l}0.78 \\
(0.65-0.93)\end{array}$ & $\begin{array}{l}0.86 \\
(0.66-1.13)\end{array}$ & $\begin{array}{l}0.77 \\
(0.6 \mathrm{I}-0.98)\end{array}$ & $\begin{array}{l}0.82 \\
(0.7 I-0.95)\end{array}$ & $\begin{array}{l}0.82 \\
(0.66-1.03)\end{array}$ & $\begin{array}{l}0.84 \\
(0.69-1.02)\end{array}$ \\
\hline Stroke & $\begin{array}{l}1.44 \\
(1.16-1.80)\end{array}$ & $\begin{array}{l}1.53 \\
(1.10-2.15)\end{array}$ & $\begin{array}{l}1.37 \\
(1.03-1.85)\end{array}$ & $\begin{array}{l}\text { I.II } \\
(0.92-1.34)\end{array}$ & $\begin{array}{l}1.15 \\
(0.86-1.54)\end{array}$ & $\begin{array}{l}\text { I.08 } \\
(0.84-1.38)\end{array}$ \\
\hline Kidney disease & $\begin{array}{l}1.13 \\
(0.89-1.43)\end{array}$ & $\begin{array}{l}0.88 \\
(0.60-1.29)\end{array}$ & $\begin{array}{l}1.32 \\
(0.97-1.79)\end{array}$ & $\begin{array}{l}1.33 \\
(1.09-1.62)\end{array}$ & $\begin{array}{l}1.45 \\
(1.05-2.01)\end{array}$ & $\begin{array}{l}1.23 \\
(0.95-1.59)\end{array}$ \\
\hline Heart failure & $\begin{array}{l}1.18 \\
(0.95-1.46)\end{array}$ & $\begin{array}{l}1.29 \\
(0.94-1.78)\end{array}$ & $\begin{array}{l}\text { I.10 } \\
(0.82-1.46)\end{array}$ & $\begin{array}{l}1.50 \\
(1.27-1.78)\end{array}$ & $\begin{array}{l}1.80 \\
(1.38-2.31)\end{array}$ & $\begin{array}{l}1.30 \\
(1.04-1.63)\end{array}$ \\
\hline \multicolumn{7}{|l|}{ Symptoms } \\
\hline Chest pain & $\begin{array}{l}0.83 \\
(0.69-1.01)\end{array}$ & $\begin{array}{l}0.83 \\
(0.63-1.10)\end{array}$ & $\begin{array}{l}0.79 \\
(0.61-1.03)\end{array}$ & $\begin{array}{l}0.68 \\
(0.59-0.79)\end{array}$ & $\begin{array}{l}0.68 \\
(0.55-0.86)\end{array}$ & $\begin{array}{l}0.67 \\
(0.55-0.82)\end{array}$ \\
\hline Generalized Weakness & $\begin{array}{l}1.33 \\
(1.11-1.59)\end{array}$ & $\begin{array}{l}1.41 \\
(1.07-1.87)\end{array}$ & $\begin{array}{l}1.32 \\
(1.05-1.68)\end{array}$ & $\begin{array}{l}1.23 \\
(1.06-1.43)\end{array}$ & $\begin{array}{l}1.16 \\
(0.91-1.48)\end{array}$ & $\begin{array}{l}1.31 \\
(1.07-1.59)\end{array}$ \\
\hline Orthopnea & $\begin{array}{l}0.88 \\
(0.74-1.05)\end{array}$ & $\begin{array}{l}0.79 \\
(0.60-1.06)\end{array}$ & $\begin{array}{l}0.98 \\
(0.77-1.24)\end{array}$ & $\begin{array}{l}1.50 \\
(1.27-1.78)\end{array}$ & $\begin{array}{l}1.80 \\
(1.38-2.31)\end{array}$ & $\begin{array}{l}1.30 \\
(1.04-1.63)\end{array}$ \\
\hline \multicolumn{7}{|l|}{ Laboratory factors } \\
\hline$<30$ & $\begin{array}{l}1.66 \\
(1.25-2.22)\end{array}$ & $\begin{array}{l}1.78 \\
(1.11-2.83)\end{array}$ & $\begin{array}{l}1.60 \\
(1.10-2.33)\end{array}$ & $\begin{array}{l}1.66 \\
(1.31-2.12)\end{array}$ & $\begin{array}{l}1.34 \\
(0.90-2.00)\end{array}$ & $\begin{array}{l}1.97 \\
(1.44-2.69)\end{array}$ \\
\hline $30-44$ & $\begin{array}{l}1.47 \\
(1.14-1.87)\end{array}$ & $\begin{array}{l}1.76 \\
(1.21-2.58)\end{array}$ & $\begin{array}{l}1.29 \\
(0.93-1.80)\end{array}$ & $\begin{array}{l}1.43 \\
(1.17-1.76)\end{array}$ & $\begin{array}{l}1.02 \\
(0.74-1.42)\end{array}$ & $\begin{array}{l}1.81 \\
(1.38-2.36)\end{array}$ \\
\hline $45-59$ & $\begin{array}{l}1.14 \\
(0.89-1.44)\end{array}$ & $\begin{array}{l}1.20 \\
(0.84-|.7|)\end{array}$ & $\begin{array}{l}1.06 \\
(0.77-1.48)\end{array}$ & $\begin{array}{l}1.17 \\
(0.97-1.42)\end{array}$ & $\begin{array}{l}1.08 \\
(0.81-1.45)\end{array}$ & $\begin{array}{l}1.22 \\
(0.94-1.59)\end{array}$ \\
\hline \multicolumn{7}{|l|}{ Systolic BP $(\mathrm{mmHg})$} \\
\hline$<100$ & $\begin{array}{l}1.50 \\
(1.03-2.19)\end{array}$ & $\begin{array}{l}1.55 \\
(0.83-2.90)\end{array}$ & $\begin{array}{l}1.38 \\
(0.86-2.24)\end{array}$ & $\begin{array}{l}1.03 \\
(0.72-1.47)\end{array}$ & $\begin{array}{l}0.94 \\
(0.52-1.70)\end{array}$ & $\begin{array}{l}1.09 \\
(0.70-1.72)\end{array}$ \\
\hline $100-119$ & $\begin{array}{l}1.03 \\
(0.7 \mid-1.49)\end{array}$ & $\begin{array}{l}\text { I.II } \\
(0.62-2.00)\end{array}$ & $\begin{array}{l}0.95 \\
(0.59-1.54)\end{array}$ & $\begin{array}{l}1.21 \\
(0.86-1.69)\end{array}$ & $\begin{array}{l}1.07 \\
(0.62-1.85)\end{array}$ & $\begin{array}{l}1.33 \\
(0.86-2.05)\end{array}$ \\
\hline $120-139$ & $\begin{array}{l}0.84 \\
(0.64-I .17)\end{array}$ & $\begin{array}{l}0.63 \\
(0.39-1.00)\end{array}$ & $\begin{array}{l}0.99 \\
(0.69-\mid .4 I)\end{array}$ & $\begin{array}{l}0.82 \\
(0.64-1.05)\end{array}$ & $\begin{array}{l}0.60 \\
(0.39-0.91)\end{array}$ & $\begin{array}{l}1.01 \\
(0.74-1.39)\end{array}$ \\
\hline $140-159$ & $\begin{array}{l}0.58 \\
(0.43-0.78)\end{array}$ & $\begin{array}{l}0.59 \\
(0.36-0.96)\end{array}$ & $\begin{array}{l}0.55 \\
(0.38-0.82)\end{array}$ & $\begin{array}{l}0.62 \\
(0.48-0.81)\end{array}$ & $\begin{array}{l}0.52 \\
(0.34-0.80)\end{array}$ & $\begin{array}{l}0.68 \\
(0.49-0.94)\end{array}$ \\
\hline$\geq 160$ & $\begin{array}{l}0.43 \\
(0.31-0.60)\end{array}$ & $\begin{array}{l}0.48 \\
(0.29-0.8 I)\end{array}$ & $\begin{array}{l}0.35 \\
(0.23-0.55)\end{array}$ & $\begin{array}{l}0.50 \\
(0.38-0.66)\end{array}$ & $\begin{array}{l}0.45 \\
(0.29-0.70)\end{array}$ & $\begin{array}{l}0.52 \\
(0.36-0.73)\end{array}$ \\
\hline
\end{tabular}

Abbreviations: BP, blood pressure; BMI, body mass index; $\mathrm{Cl}$, confidence interval; COPD, chronic obstructive pulmonary disease; eGFR, estimated glomerular filtration rate; $\mathrm{OR}$, odds ratio. 
study showed encouraging improvements in the likelihood of surviving at both 3 months and 1 year in patients discharged from all metropolitan Worcester hospitals after acute HF in 2000 as compared with 1995.

Although the observational nature of the investigation does not allow for proof of causality, the observed improvements in long-term prognosis may be due to the improved hospital treatment of acute HF, increased use and effectiveness of outpatient clinics for patients with HF, greater use of effective medical and nonpharmacologic regimens for hospital survivors of HF and greater adherence to these treatment modalities, and/or increased provider attention to the use of guidelines for the more effective management of patients with HF.

Few studies have described the long-term prognosis associated with acute HF from a more generalizable communitybased perspective or determined whether or not the long-term prognosis of patients with HF has changed over time. In a large population-based study of Olmsted County, Minnesota, residents, the 5-year age-adjusted survival rates associated with HF improved significantly between 1991 and $1996 .^{15}$ In an investigation of more than 1000 Framingham Heart Study enrollees who had developed new-onset HF between 1950 and 1999, improvements in survival after new-onset HF were estimated to be approximately $12 \%$ per decade for men and women enrolled in this seminal longitudinal study. ${ }^{16}$ Improving trends in the 3-year survival rates after new-onset HF have also been demonstrated in a national study of older and younger patients from Scotland with HF over the period 1986-1995. ${ }^{17}$ In a prior analysis of data collected in the Worcester Heart Failure Study, the authors of the present study described the long-term death rates associated with acute HF in residents of the Worcester metropolitan area discharged after acute HF in $2000{ }^{8}$ The present study extends the findings of these community-based studies to a relatively contemporary and high-risk cohort of predominantly elderly patients hospitalized for acute HF.

The data suggest that HF remains a highly serious and lethal condition for most hospitalized patients but that patients' long-term prognosis has been improving. The authors hypothesize that increases in the percentage of patients receiving cardiac medications shown to be of benefit in improving the long-term prognosis of patients with HF over the years under study may have contributed to the improvements in long-term prognosis noted between 1995 and 2000, though this hypothesis was unable to be directly tested due to the nonrandomized observational nature of the present investigation.

\section{Factors associated with an increased risk of dying after hospital discharge for HF}

To better understand the factors affecting prognosis after $\mathrm{HF}$, particularly for patients encountered in the broader community setting, the independent prognostic significance of several demographic and clinical factors on long-term survival at different time points was evaluated. At 3 months after hospital discharge, older age, a lower blood pressure and BMI, and the presence of previously diagnosed COPD, chronic kidney disease, stroke, and prior HF were associated with an increased risk of dying.

Similar prognostic variables have been identified in other studies evaluating short-term mortality among patients with $\mathrm{HF}^{18,19}$ The association between COPD and HF is well known, as between $10 \%$ and $50 \%$ of all HF patients also have coexisting COPD. ${ }^{20}$ In a similar fashion to HF, COPD is characterized by both recurrent acute exacerbations as well as by a general progression of disease over time..$^{21}$ In addition, the stress on the cardiovascular system from complications of COPD, including hypoxia, cor pulmonale, and polycythemia, could theoretically exacerbate HF over time, and the presence of COPD may make it more difficult to diagnose $\mathrm{HF}^{20}$ Furthermore, beta blockers may not be tolerated by patients with both COPD and HF, and beta agonist treatment of COPD has been associated with increased mortality in these individuals. $^{22}$

Previous studies have found that approximately onethird of patients with HF will also have concomitant renal dysfunction, ${ }^{23}$ and as with COPD, the relationship between chronic and acute renal disease and adverse prognosis has been well documented. ${ }^{24}$ Renal dysfunction complicates acute and chronic treatment decisions in volume-overloaded patients, and renal function may be worsened by concomitant diuretic administration. ${ }^{25}$

The data of this present study suggest that early mortality after hospitalization for HF is strongly associated with advanced age, a previous history of $\mathrm{HF}$, and the presence of other serious comorbid conditions, several of which appear to be secondary to chronic HF such as cardiorenal syndrome. Relatively similar factors were associated with an increased risk of dying during the first year after discharge. As has been noted in prior studies involving patients with acute HF, BMI was inversely related to mortality in the present study's hospitalized cohort. ${ }^{26,27}$ It has been hypothesized that cardiac cachexia may contribute to the "obesity paradox" in $\mathrm{HF}$, although the causes of cachexia among patients with severe HF are complex and incompletely understood. ${ }^{28}$ 
It also remains unclear whether patients who are obese are more likely to develop or be diagnosed with acute HF earlier and the extent to which obesity affects prognosis once HF is manifest.

Factors associated with a poor prognosis after hospital discharge for acute HF, including those associated with preserved versus systolic HF, merit further study, particularly with regards to better understanding the possible mechanisms involved so that appropriate advice and treatment regimens may be prescribed for patients with acute HF and its major subtypes.

Although considerable advances have been made and will continue to be made in the management of patients with acute $\mathrm{HF}$, it is important that clinicians have a realistic understanding of the long-term prognosis associated with this clinical syndrome, including the extremely high death rates observed in the present study. Clinicians also need to be aware of factors that adversely affect patients' long-term survival, which may facilitate targeted treatment toward specific high-risk groups or help inform decisions about making realistic expectations about prognosis. Decisions about the use of more aggressive interventions for patients with HF must also take into account the presence and effect of various comorbidities on the patient's long-term survival outlook.

In the present study, post-discharge decedents were less likely to be discharged on effective cardiac medications, perhaps due to the fact that these patients were elderly, more likely to be hypotensive, and had worse renal function at the time of hospitalization compared with those who survived. In light of the fact that elderly patients tend to have more comorbid conditions, many of which also preclude the use of medications known to improve long-term survival, and since they may be less likely to be prescribed these medications due to their advanced age, it is not surprising that their prognosis is worse.

A recent meta-analysis examining the efficacy of ACE inhibitors in patients with chronic HF with preserved ejection fraction found that ACE inhibitors reduced HF-related hospitalizations in elderly patients. ${ }^{29}$ Another meta-analysis of more than 12,000 patients with systolic HF found that $\beta$-blockers improved mortality in elderly patients, with a similar relative risk reduction in mortality between elderly and nonelderly patients. ${ }^{30}$ The aldosterone antagonists are another important class of medications shown to have benefit in patients with systolic HF. ${ }^{31}$ Despite the known benefits associated with the use of these medications, they remain underprescribed in patients discharged from the hospital after decompensated HF, particularly in elderly patients. ${ }^{32}$ Treating these high-risk patients with evidence-based therapies to optimize their long-term survival, while balancing risk, remains a clinical challenge and an area in need of further investigation.

\section{Study strengths and limitations}

The primary strength of this study is the inclusion of a large sample of patients hospitalized with independently validated HF from a well characterized New England metropolitan area. In addition, information was collected about a number of different factors that could affect the long-term prognosis after HF, and the rates of patient follow-up were excellent. The limitations of the study include the inability to systematically examine the long-term prognosis of patients with acute HF according to type of left ventricular dysfunction (systolic versus diastolic) given the extent of missing data with regard to ejection fraction findings. Also, information on cause-specific mortality from death certificates was not collected. In addition, it was not possible to assess the prognostic value of brain natriuretic peptide and troponin laboratory values as these factors were not routinely measured at the greater Worcester medical centers studied in 1995 and 2000.

\section{Conclusion}

The results of the present study suggest that the long-term survival outlook for patients discharged from the hospital after acute HF remains poor but is improving. A number of characteristics associated with a poor long-term prognosis were identified, several of which might be effectively intervened upon. The present results provide further impetus for current efforts to improve the management of patients with acute HF and for the further development and increased use of long-term strategies to enhance patients' adherence to effective medical and nonpharmacologic treatment regimens.

\section{Acknowledgments}

This study was made possible through the cooperation of the administration, medical records, and cardiology departments of participating Worcester metropolitan area hospitals. Funding support for this study was provided by the National Heart, Lung, and Blood Institute (R37 HL69874 and 1U01HL105268-01).

\section{Disclosure}

The authors report no conflicts of interest in this work. 


\section{References}

1. Hunt SA, Abraham WT, Chin MH, et al. 2009 focused update incorporated into the ACC/AHA 2005 Guidelines for the Diagnosis and Management of Heart Failure in Adults: a report of the American College of Cardiology Foundation/American Heart Association Task Force on Practice Guidelines: developed in collaboration with the International Society for Heart and Lung Transplantation. Circulation. 2009;119:e391-e479.

2. Rosamond W, Flegal K, Furie K, et al. Heart disease and stroke statistics - 2008 update: a report from the American Heart Association Statistics Committee and Stroke Statistics Subcommittee. Circulation. 2008;117:e25-e146.

3. Abdel-Qadir HM, Tu JV, Yun L, et al. Diuretic dose and long-term outcomes in elderly patients with heart failure after hospitalization. Am Heart J. 2010;160:264-271. e1.

4. Kociol RD, Greiner MA, Hammill BG, et al. Long-term outcomes of Medicare beneficiaries with worsening renal function during hospitalization for heart failure. Am J Cardiol. 2010;105:1786-1793.

5. Harjola VP, Follath F, Nieminen MS, et al. Characteristics, outcomes, and predictors of mortality at 3 months and 1 year in patients hospitalized for acute heart failure. Eur J Heart Fail. 2010;12:239-248.

6. Jhund PS, Macintyre K, Simpson CR, et al. Long-term trends in first hospitalization for heart failure and subsequent survival between 1986 and 2003: a population study of 5.1 million people. Circulation. 2009;119:515-523.

7. Shafazand M, Schaufelberger M, Lappas G, et al. Survival trends in men and women with heart failure of ischaemic and non-ischaemic origin: data for the period 1987-2003 from the Swedish Hospital Discharge Registry. Eur Heart J. 2009;30:671-678.

8. Goldberg RJ, Ciampa J, Lessard D, et al. Long-term survival after heart failure: a contemporary population-based perspective. Arch Intern Med. 2007; 167:490-496.

9. Goldberg RJ, Spencer FA, Farmer C, Meyer TE, Pezzella S. Incidence and hospital death rates associated with heart failure: a community-wide perspective. Am J Med. 2005;118:728-734.

10. Goldberg RJ, Darling C, Joseph B, et al. Epidemiology of decompensated heart failure in a single community in the northeastern USA. Am J Cardiol. 2009;104:377-382.

11. McKee PA, Castelli WP, McNamara PM, et al. The natural history of congestive heart failure: the Framingham Study. $N$ Engl J Med. 1971;285:1441-1446.

12. Levey AS, Bosch JP, Lewis JB, Greene T, Rogers N, Roth D. A more accurate method to estimate glomerular filtration rate from serum creatinine: a new prediction equation. Modification of diet in renal disease study group. Ann Intern Med. 1999;130:461-470.

13. Goldberg RJ, Spencer FA, Farmer C, Lessard D, Pezzella SM, Meyer TE. Use of disease modifying therapies in patients hospitalized with heart failure: a population-based perspective. Am J Med. 2007;120: 98. e1-e8.

14. Metra M, Gheorghiade M, Bonow RO, Cas LD. Postdischarge assessment after a heart failure hospitalization: the next step forward. Circulation. 2010;122:1782-1785.

15. Senni M, Tribouilloy CM, Rodeheffer RJ, et al. Congestive heart failure in the community: a study of all incident cases in Olmsted County, Minnesota, in 1991. Circulation. 1998;98:2282-2289.

Clinical Epidemiology

\section{Publish your work in this journal}

Clinical Epidemiology is an international, peer-reviewed, open access journal focusing on disease and drug epidemiology, identification of risk factors and screening procedures to develop optimal preventative initiatives and programs. Specific topics include: diagnosis, prognosis, treatment, screening, prevention, risk factor modification, systematic
16. Levy D, Kenchaiah S, Larson MG, et al. Long-term trends in the incidence of and survival with heart failure. N Engl J Med. 2002;347: 1397-1402.

17. MacIntyre K, Capewell S, Stewart S, et al. Evidence of improving prognosis in heart failure: trends in case fatality in 66547 patients hospitalized between 1986 and 1995. Circulation. 2000;102:1126-1131.

18. Lassus JP, Nieminen MS, Peuhkurinen K, et al. Markers of renal function and acute kidney injury in acute heart failure: definitions and impact on outcomes of the cardiorenal syndrome. Eur Heart J. 2010;31:2791-2798.

19. Harjola VP, Follath F, Nieminen MS, et al. Characteristics, outcomes, and predictors of mortality at 3 months and 1 year in patients hospitalized for acute heart failure. Eur J Heart Fail. 2010;12:239-248.

20. Hawkins NM, Petrie MC, Jhund PS, Chalmers GW, Dunn FG, McMurray JJ. Heart failure and chronic obstructive pulmonary disease: diagnostic pitfalls and epidemiology. Eur J Heart Fail. 2009;11:130-139.

21. Pauwels RA, Rabe KF. Burden and clinical features of chronic obstructive pulmonary disease (COPD). Lancet. 2004;364:613-620.

22. Au DH, Udris EM, Curtis JR, McDonell MB, Fihn SD. Association between chronic heart failure and inhaled beta-2-adrenoceptor agonists. Am Heart J. 2004;148:915-920.

23. Adams KF Jr, Fonarow GC, Emerman CL, et al. Characteristics and outcomes of patients hospitalized for heart failure in the United States: rationale, design, and preliminary observations from the first 100,000 cases in the Acute Decompensated Heart Failure National Registry (ADHERE). Am Heart J. 2005;149:209-216

24. Smith GL, Lichtman JH, Bracken MB, et al. Renal impairment and outcomes in heart failure: systematic review and meta-analysis. $J \mathrm{Am}$ Coll Cardiol. 2006;47:1987-1996.

25. Lazzarini V, Bettari L, Bugatti S, et al. Can we prevent or treat renal dysfunction in acute heart failure? Heart Fail Rev. Epub May 10, 2011.

26. Horwich TB, Fonarow GC, Hamilton MA, MacLellan WR, Woo MA, Tillisch JH. The relationship between obesity and mortality in patients with heart failure. J Am Coll Cardiol. 2001;38:789-795.

27. Curtis JP, Selter JG, Wang Y, et al. The obesity paradox: body mass index and outcomes in patients with heart failure. Arch Intern Med. 2005;165:55-61.

28. Davos $\mathrm{CH}$, Doehner W, Rauchhaus M, et al. Body mass and survival in patients with chronic heart failure without cachexia: the importance of obesity. J Card Fail. 2003;9:29-35.

29. Fu M, Zhou J, Sun A, et al. Efficacy of ACE inhibitors in chronic heart failure with preserved ejection fraction - a meta analysis of 7 prospective clinical studies. Int J Cardiol. Epub April 9, 2011.

30. Dulin BR, Haas SJ, Abraham WT, Krum H. Do elderly systolic heart failure patients benefit from beta blockers to the same extent as the non-elderly? Meta analysis of $>12,000$ patients in large scale clinical trials. Am J Cardiol. 2005;95:896-898.

31. Zannad F, McMurray JJ, Krum H, et al. Eplerenone in patients with systolic heart failure and mild symptoms. N Engl J Med. 2011;364: $11-21$.

32. Albert NM, Yancy CW, Liang L, et al. Use of aldosterone antagonists in heart failure. JAMA. 2009;302:1658-1665.

reviews, risk \& safety of medical interventions, epidemiology \& biostatical methods, evaluation of guidelines, translational medicine, health policies \& economic evaluations. The manuscript management system is completely online and includes a very quick and fair peer-review system, which is all easy to use. 